\title{
Utilization of a Linear Solver for Multiscale Design and Optimization of Microstructures
}

\author{
Pinar Acar* and Veera Sundararaghavan $\ddagger$ \\ University of Michigan, Ann Arbor, Michigan 48109
}

DOI: $\underline{10.2514 / 1 . J 054822}$

\begin{abstract}
Microstructures have a significant effect on the performance of critical components in numerous aerospace metallic material applications. Examples include panels in airframes that are exposed to high temperatures and sensors used for vibration tuning. This paper addresses the techniques to optimize the microstructure design for polycrystalline metals. The microstructure is quantified with the orientation distribution function that determines the volume densities of crystals that make up the polycrystal microstructure. The orientation distribution function of polycrystalline alloys (e.g., hexagonal close-packed titanium) is represented in a discrete form, and the volumeaveraged properties are computed through the orientation distribution function. The optimization is performed using the space of all possible volume-averaged macroproperties (stiffness and thermal expansion). A direct linear solver is employed to find the optimal orientation distribution functions. The direct solver is capable of finding exact solutions even for problems with multiple or infinite solutions. It is first applied to the optimization of the panel-buckling problem. The objective of the buckling optimization problem is to find the best microstructure design that maximizes the critical buckling temperature. The optimum solution computed with this approach is found to be same as the optimum solution of a global approach that uses a genetic algorithm. The linear solver methodology is extended to plastic properties and applied to explore the design of a Galfenol beam microstructure for vibration tuning with a yielding objective. The design approach is shown to lead to multiple optimum solutions.
\end{abstract}

\section{Introduction}

$\mathbf{M}$ ANY aerospace applications use materials that operate at high temperatures. For example, titanium panels in high-speed vehicles are exposed to elevated temperatures. The microstructure of such alloys has an important effect on the performance under high thermal stresses. Thus, the optimization of the microstructure is expected to significantly improve the performance. Response of plate panels in compression, due to thermal loading and edge restraints, is a classical plate-buckling problem. The stability analysis of isotropic metal plates is a general problem and can be found in textbooks [1]. However, the isotropy assumption is not justified when including microstructural effects and is in direct conflict with the trends in new materials development, where one of the major goals is to enhance the properties of the material in certain directions while sacrificing the properties in other directions where they are not as important (e.g., development of laminated composite systems). Likewise, techniques that allow tailoring of properties of polycrystalline alloys (e.g., Ti, $\mathrm{Al}$ used in structural panels) involve tailoring of preferred orientations of various crystals constituting the polycrystalline alloy (see Fig. 1). Using alloys with tailored crystallite distribution, this paper addresses the problem of optimizing properties in critical applications.

The microstructure is quantified using the orientation distribution function (ODF). The ODF represents the volume fractions of the crystals of different orientations in the microstructure. The ODF is defined based on a parameterization of the crystal lattice rotation. Popular representations include Euler angles [2,3] and classes of angle-axis representations, with the most popular being the

Presented as Paper 2016-0156 at the 57th AIAA/ASCE/AHS/ASC Structures, Structural Dynamics, and Materials Conference, San Diego, CA, 4-8 January 2016; received 14 October 2015; revision received 14 December 2015; accepted for publication 14 December 2015; published online 25 February 2016. Copyright $\odot 2015$ by Pinar Acar. Published by the American Institute of Aeronautics and Astronautics, Inc. All rights reserved. Copies of this paper may be made for personal and internal use, on condition that the copier pay the per-copy fee to the Copyright Clearance Center (CCC). All requests for copying and permission to reprint should be submitted to $\mathrm{CCC}$ at www.copyright.com; employ the ISSN 0001-1452 (print) or 1533-385X (online) to initiate your request.

*Graduate Research Assistant, Department of Aerospace Engineering. Student Member AIAA.

${ }^{\dagger}$ Associate Professor, Department of Aerospace Engineering. Member AIAA.
Rodrigues parameterization [4]. Conversion of continuous orientation space to finite degrees of freedom for material property optimization requires discretization techniques. Discretization schemes either focus on a global basis (e.g., Fourier space or spherical harmonics $[5,6])$ or a local basis using a finite element discretized Rodrigues space with polynomial shape functions defined locally over each element $[\underline{7}, \underline{8}]$. Liu et al. [9] identified the ODF solutions to various optimization problems by directly sampling the ODF space using a data mining methodology. However, sampling in the property space is favorable because the number of design variables is significantly lesser. The microstructure-sensitive design methodology presented by Fast et al. [10] employs sampling within the property hull similar to this work. However, the discretization scheme for the ODF was based on a Fourier basis. The Fourier/ spectral methods cannot represent sharp textures due to the use of a global basis. In contrast, the finite element approach presented here can lead to single-crystal solutions (or sharp textures) due to the use of a local basis. In this paper, we demonstrate, for the first time, the generation of multiple polycrystal solutions in an engineering optimization problem using the finite element representation of the ODF.

The objective of the present work is to find out the optimal ODFs that maximize/minimize a macroscale objective function. Because the number of unknowns in the ODF is large, the optimization is first performed in the space of all possible material properties. When a lower- or upper-bound homogenization approach is employed, the space of all possible properties is simply a convex hull with the vertices represented by single-crystal properties. Once the best set of properties is found, the best microstructure (ODF) corresponding to that property can be identified using linear programming. To demonstrate the approach, the optimal ODFs that maximize the critical increase in the buckling temperature of a simply supported hexagonal close-packed (HCP) titanium plate are identified. The optimum result is compared to the results from a global approach that uses a genetic algorithm with sampling in the complete ODF space. Another example is shown where the optimization methodology is extended to a problem involving vibration tuning of a Galfenol beam. We show that the approach can lead us to multiple ODF solutions. The remainder of this paper is organized as follows. In Sec. II, the analytical solution of the simply supported thermal buckling problem is introduced. Section III addresses the computation of microstructure properties using an orientation distribution function. Section IV addresses the optimization methodology, utilization of the linear solver, optimization results, and 


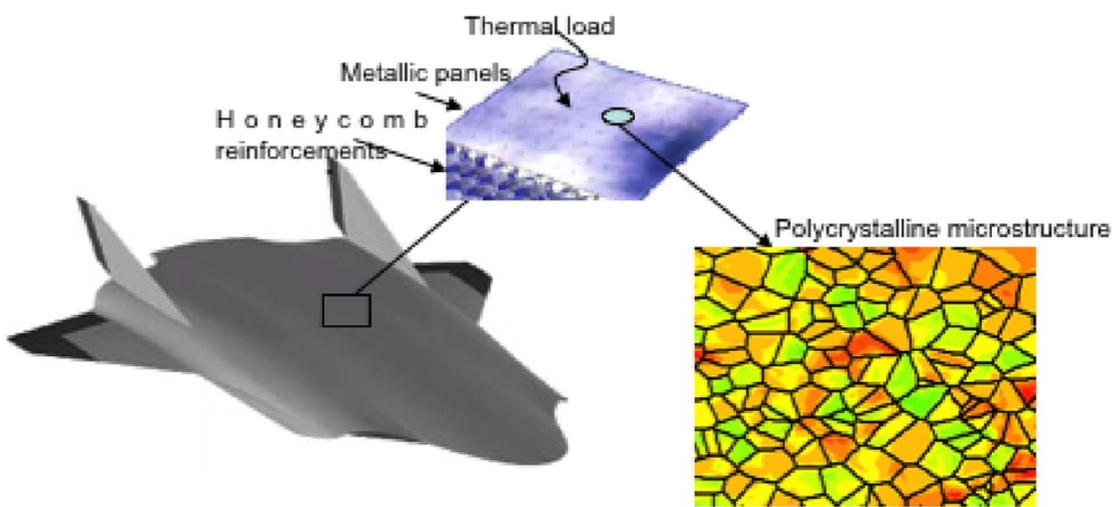

Fig. 1 Microstructure of high-temperature materials.

their comparison with the global approach. In Sec. V, we discuss the Galfenol beam vibration-tuning problem with multiple optimum solutions. The summary of the paper and the potential extensions are discussed in Sec. VI.

\section{Critical Increase in Temperature for Buckling of a Plate Under Uniform Heating}

Airframe panels made of titanium alloys can be modeled as thin, rectangular, anisotropic plates. In this example, we include full anisotropy of the thermal expansion tensor but restrict the stiffness to be orthotropic. The thermal buckling problem of such plates (when simply supported) has been solved analytically [11]. The principal directions are taken to be parallel to the sides, and the material is compressed by uniformly distributed axial load $N_{x}$ (Fig. 2). The representation of one of the meshes used for this problem is shown in Fig. 3 .

The deflection equation of the orthotropic plate will have the form

$$
D_{11} \frac{\partial^{4} w}{\partial x^{4}}+2 D_{33} \frac{\partial^{4} w}{\partial x^{2} \partial y^{2}}+D_{22} \frac{\partial^{4} w}{\partial y^{4}}+N_{x} \frac{\partial^{2} w}{\partial x^{2}}+N_{y} \frac{\partial^{2} w}{\partial y^{2}}=0
$$

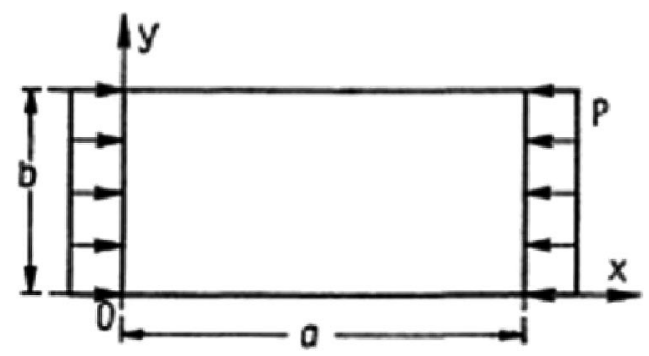

Fig. 2 Representation of the analytical problem.

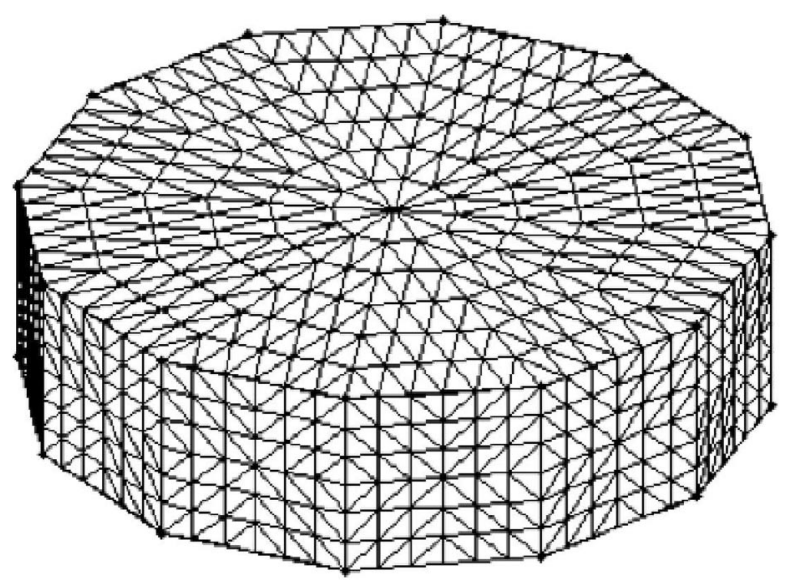

Fig. 3 Mesh 2 representation. where $D$ values are the flexural rigidities that are computed using the stiffness tensor components $\left(C_{i j}\right)$ :

$$
\begin{aligned}
& {\left[\begin{array}{lll}
D_{11} & D_{12} & D_{13} \\
D_{21} & D_{22} & D_{23} \\
D_{31} & D_{32} & D_{33}
\end{array}\right]=\frac{h^{3}}{12} \times\left(\left[\begin{array}{lll}
C_{11} & C_{12} & C_{16} \\
C_{21} & C_{22} & C_{26} \\
C_{61} & C_{62} & C_{66}
\end{array}\right]\right.} \\
& -\left[\begin{array}{lll}
C_{13} & C_{14} & C_{15} \\
C_{23} & C_{24} & C_{25} \\
C_{63} & C_{64} & C_{65}
\end{array}\right] \times\left(\left[\begin{array}{lll}
C_{33} & C_{34} & C_{35} \\
C_{43} & C_{44} & C_{45} \\
C_{53} & C_{54} & C_{55}
\end{array}\right]\right)^{-1} \\
& \left.\times\left[\begin{array}{lll}
C_{31} & C_{32} & C_{36} \\
C_{41} & C_{42} & C_{46} \\
C_{51} & C_{52} & C_{56}
\end{array}\right]\right)
\end{aligned}
$$

where $h$ is the thickness of the plate. The solution series for the plate deflection in the $z$ direction are given next:

$$
w=A_{\mathrm{mn}} \sin \left(\frac{m \pi x}{a}\right) \sin \left(\frac{n \pi y}{b}\right)
$$

where $A_{m n}$ are constants, and $m$ and $n$ are integers. This case employs a simply supported orthotropic plate with the given boundary conditions:

$$
w=0, \quad \frac{\partial w^{2}}{\partial x^{2}}+\nu_{y} \frac{\partial w^{2}}{\partial y^{2}}=0 \quad \text { at } x=0, a
$$

$$
w=0, \quad \frac{\partial w^{2}}{\partial y^{2}}+\nu_{x} \frac{\partial w^{2}}{\partial x^{2}}=0 \quad \text { at } y=0, b,
$$

where $\nu_{x}$ and $\nu_{y}$ are Poisson's ratios. By requiring that Eq. (3) be a solution of Eq. (1), the relation can be obtained:

$$
N_{x}\left(\frac{m}{a}\right)^{4}+N_{y}\left(\frac{n}{b}\right)^{4}=\pi^{2}\left[D_{11} \frac{m}{a}+2 D_{33} \frac{m n}{a b}+D_{22} \frac{n}{b}\right]
$$

This formulation here will be given for a general case where forces $N_{x}$ and $N_{y}$ may vary but must maintain a constant ratio $\beta$. However, the problem of interest in this work only has $N_{x}$ as the axial force (Fig. 2), i.e., $\beta=0$ :

$$
N_{x}=\lambda, \quad N_{y}=\lambda \beta
$$




$$
\begin{aligned}
& \lambda=\frac{\pi^{2} \sqrt{D_{11} D_{22}}}{b^{2}} \\
& \cdot \frac{\sqrt{D_{11} / D_{22}}(m / c)^{2}+\left(2 D_{33} / \sqrt{D_{11} D_{22}}\right) n^{2}+\sqrt{D_{22} / D_{11}}(c / m)^{2} n^{4}}{1+\beta(c / m)^{2} n^{2}}
\end{aligned}
$$

where $c$ is the ratio between the lengths of the sides of the plate $(c=a / b)$. The problem then consists of seeking the values of $m$ and $n$ that give the smallest $\lambda$ and hence the critical distributed buckling load $\lambda_{\mathrm{cr}}$. Based on the solution of the critical distributed buckling load, we can get the expression for the critical increase in temperature using the coefficient of thermal expansion tensor $(\alpha)$ :

$$
\Delta T_{\mathrm{cr}}=\frac{\lambda}{12 / h^{2}\left(D_{11} \cdot \alpha_{x}+D_{12} \cdot \alpha_{y}+D_{13} \cdot \alpha_{x y}\right)}
$$

The material properties $\alpha$ and $C$ are the material properties computed from the microstructure (represented using the ODF).

\section{Modeling Properties of Hexagonal Close-Packed Titanium Microstructure}

The alloy microstructure consists of multiple crystals, with each crystal having an orientation. The generalized Hooke's law for the aggregate of crystals may be written in the form:

$$
<\epsilon_{i j}>=S_{i j k l}^{\mathrm{eff}}<\sigma_{k l}>+\alpha_{i j}^{\mathrm{eff}} \Delta T
$$

where $\left\langle\epsilon_{i j}>\right.$ and $<\sigma_{k l}>$ are the volume-averaged strain and stress, respectively; $S^{\text {eff }}\left(=\left[C^{\text {eff }}\right]^{(-1)}\right)$ is the effective compliance tensor in the coordinate system of the part; $C^{\mathrm{eff}}$ is the effective stiffness tensor; and $\alpha^{\text {eff }}$ is the effective thermal-expansion tensor. Assuming homogeneity of the deformation in a macroscale elementary volume, the effective thermoelastic properties may be found through averaging using the Taylor approximation [12]:

$$
C^{\mathrm{eff}}=<C>, \quad \alpha^{\mathrm{eff}}=<\alpha>
$$

where $C$ and $\alpha$ are the stiffness and thermal expansion tensors for each crystal, and the average is done over crystals of all orientations in the microstructure. If the effect of factors (e.g., crystal size and shape) is ignored, averaging (denoted by $\langle$.$\rangle in the preceding equation)$ can be performed over the orientation distribution function (ODF, represented by $A$ ). The ODF gives the volume density of each orientation in the microstructure. If the orientation-dependent property for single crystals, $\chi(r)$, is known, any polycrystal property can be expressed as an expected value, or average, given by

$$
<\chi>=\int_{R} \chi(r) A(r, t) \mathrm{d} v
$$

where the ODF, $A$, is a function of orientation $r$ and time $t$ (for plasticity problems). The average value is computed by integrating in the representative volume element, $d v$, which can be obtained by considering the crystallographic symmetries.

The present work employs Rodrigues angle-axis parameterization of the orientation space [7] because angle-axis representations define an alternate way of representing orientations compared to Euler angles $[\underline{2}, \underline{13}]$. Rodrigues's parameterization is created by scaling the axis of rotation $n$ as $r=n \tan (\theta / 2)$, where $\theta$ is the rotation angle. Finite element discretization of the orientation space and associated integration schemes using Gauss quadrature allows matrix representation of Eq. (11). The ODF is discretized into $N$ independent nodes, with $\overline{N_{\text {elem }}}$ finite elements and $N_{\text {int }}$ integration points per element. Two different meshes, accounting for hexagonal symmetry, were used in this work to solve the problem. Mesh 1 contains 111 nodes and 288 elements. This translates to 288 integration points (one integration point per element) and 50 independent nodes (50 ODFs). However, mesh 2 includes 605 nodes and 2304 elements. This translates to 2304 integration points (one integration point per element) and 388 independent nodes (388 ODFs).

Using this parameterization, any polycrystal property can be expressed in a linear form as follows [14]:

$$
\begin{aligned}
<\chi> & =\int_{R} \chi(r) A(r, t) \mathrm{d} v \\
& =\sum_{n=1}^{N_{\text {elem }}} \sum_{m=1}^{N_{\text {int }}} \int_{R} \chi\left(r_{m}\right) A\left(r_{m}\right) w_{m}\left|J_{n}\right| \frac{1}{\left(1+r_{m} \cdot r_{m}\right)^{2}}
\end{aligned}
$$

where $A\left(r_{m}\right)$ is the value of the ODF at the $m$ th integration point with global coordinate $r_{m}$ of the $n$th element; $\left|J_{n}\right|$ is the Jacobian determinant of the $n$th element; $w_{m}$ is the integration weight associated with the $m$ th integration point; and $1 /\left(1+r_{m} \cdot r_{m}\right)^{2}$ represents the metric of Rodrigues parameterization. This is equivalent to an equation linear in the ODF: $\left\langle\chi>=p^{\text {int }^{T}} A^{\text {int }}\right.$, where $p_{l}^{\text {int }}=\chi\left(r_{i}\right) w_{i}\left|J_{i}\right| 1 /\left(1+r_{i} \cdot r_{i}\right)^{2}$ and $A^{\text {int }}=A\left(r_{i}\right), i=1, \ldots, N_{\text {int }} \times N_{\text {elem }}$.

The polycrystal stiffness $\bar{C}$ and the thermal expansion coefficient $\bar{\alpha}$ are computed through a weighted average (over $A$ ) of the stiffnesses and thermal expansion coefficients of individual crystals expressed in the sample reference frame. In a general case, use of Eq. (10) to compute the effective stiffness and thermal expansion tensor for use in the buckling model results in an anisotropic stiffness and thermal expansion tensor. The yield stress is computed using a crystal plasticity model from our recent work [9].

\section{Optimization Method}

The optimization of the panel-buckling problem is performed to find out the best microstructure design that maximizes the critical increase in buckling temperature. This section discusses optimization methodology, utilization, and test of a linear solver as well as optimization results for the buckling problem and comparison with the global approach.

For the sample dimensions of $0.25 \times 0.25 \times 0.005 \mathrm{~m}$, the $\Delta T_{\mathrm{cr}}$ of an alpha-titanium plate with optimal ODF was identified. The optimization problem enforces three separate constraints on the ODF that is desired: symmetry, normalization, and positiveness. The procedure for optimization is described here.

\section{A. Symmetry}

First, the symmetry of the HCP crystal structure is enforced by using only the "independent nodes" in the ODF mesh. Independent nodal points are the reduced set of nodes obtained by accounting for symmetry conditions at the boundaries of the ODF mesh. Let $\boldsymbol{H}$ be the matrix converting the independent nodal values $\boldsymbol{A}^{\text {node }}$ to the integration point values $\boldsymbol{A}^{\text {int }}$ through the shape functions, then $\boldsymbol{A}^{\text {int }}=\boldsymbol{H} \boldsymbol{A}^{\text {node }}$. The vector containing the values of the ODF at independent nodal points $\boldsymbol{A}^{\text {node }}$ is sufficient to describe the ODF and is hereafter referred to as $\boldsymbol{A}$.

Remark: Properties are specified using the modified $\boldsymbol{p}^{T}=\boldsymbol{p}^{\mathrm{int}^{T}} \boldsymbol{H}$ as $<\chi>=\boldsymbol{p}^{T} \boldsymbol{A}$. For calculating more than one property, $\boldsymbol{p}$ is written in a matrix form. Using reduced integration with one integration point per element at local coordinates of $(0.25,0.25,0.25)$ and an integration weight of $\boldsymbol{w}=1 / 6$, the $\boldsymbol{H}$ matrix can be defined from the equation $\boldsymbol{A}_{\boldsymbol{e}}^{\mathrm{int}}=0.25 \sum_{i=1}^{4} \boldsymbol{A}_{e}^{i}$, where $\boldsymbol{A}_{e}^{\mathrm{int}}$ is the integration point ODF value at element $\boldsymbol{e}$, and $\boldsymbol{A}_{e}^{i}, \boldsymbol{i}=1, \ldots, 4$ refers to the ODF values at the four nodes of the tetrahedral element $\boldsymbol{e}$.

\section{B. Normalization}

The constraint that the volume fractions sum to 1 is given by the following relationship:

$$
\int_{R} \operatorname{Adv}=\sum_{n=1}^{N_{\text {elem }}} \sum_{m=1}^{N_{\text {int }}} A\left(r_{m}\right) w_{m}\left|J_{n}\right| \frac{1}{\left(1+r_{m} \cdot r_{m}\right)^{2}}=1
$$


This is equivalent to the linear constraint: $\boldsymbol{q}^{\text {int }}{ }^{T} \boldsymbol{A}^{\text {int }}=1$ where $\boldsymbol{q}_{i}^{\text {int }}=\boldsymbol{w}_{i}\left|\boldsymbol{J}_{i}\right| \frac{1}{\left(1+\boldsymbol{r}_{i} \cdot \boldsymbol{r}_{i}\right)^{2}}$ and $\boldsymbol{A}_{i}^{\text {int }}=\boldsymbol{A}\left(\boldsymbol{r}_{i}\right)$, where each $\boldsymbol{i}$ corresponds to a combination of $(\boldsymbol{n}, \boldsymbol{m}), \boldsymbol{i}=1, \ldots, \boldsymbol{N}_{\text {int }} \times \boldsymbol{N}_{\text {elem }}$. This can also be written in terms of independent nodes as $\boldsymbol{q}^{T}=\boldsymbol{q}^{\text {int }^{T}} \boldsymbol{H}$ as $\boldsymbol{q}^{T} \boldsymbol{A}=\mathbf{1}$.

\section{Positiveness}

The positivity of the ODF is enforced by the constraint $\boldsymbol{A} \geq 0$ (i.e., the volume fractions are positive).

Mathematical representation of the optimization problem is given next:

$$
\max \Delta T_{\text {cr }}
$$

$$
\text { subject to } q^{T} A=1, \quad A \geq 0
$$

\section{Utilization of a Direct Linear Solver to the Optimization Problem}

The optimization is performed in a solution space that is reduced to the space of stiffness and thermal expansion parameters from the complete ODF space. This reduction saves a great amount of computational time because the complete ODF space has 50 parameters for mesh 1 and 388 parameters for mesh 2. However, the macroscopic property space has 12 parameters (nine independent $C$ elements for orthotropic stiffness modeling and three independent $\alpha$ parameters for anisotropic thermal expansion modeling) for both meshes.

Property closures represent the complete range of properties obtainable from the space of ODFs. These are approximated by the space between upper and lower bounds of the given property. Upperbound closure of stiffness values represents the range of properties obtainable by the upper-bound homogenization relation in Eq. (17). The hull maps the full range of upper-bound values of a combination of stiffness values. The extremal textures were found to correspond to single crystals. A simple technique for constructing property closures (for the homogenization relations considered here) is by establishing the smallest convex region enveloping single-crystal property points. Linear programming, although more rigorous, is more intuitive for construction of property closures because closures are obtained as a result of property maximization or minimization. Connecting faces on the closure may contain polycrystals that are explicitly identified by the LP approach. This approach is also well suited for other problems, such as identification of textures with desired property combinations where several properties are optimized simultaneously. Let $v_{1}, v_{2}$ be the set of properties for which the closure is required. The closure for property $v_{1}$ is first found by obtaining the extremal values $\left(v_{1 \mathrm{max}}, v_{1 \mathrm{~min}}\right)$. Then, property $v_{1}$ is discretized into $m$ values $v_{1}^{i}, i=1, . ., m$ between $v_{1 \max }$ and $v_{1 \text { min }}$. The property closure of the combined set of properties $\left(v_{1}, v_{2}\right)$ is found by executing a similar extremum LP problem at each point $v_{1}^{i}$ with the additional constraint that $\boldsymbol{p}_{1}^{T} \boldsymbol{A}=\boldsymbol{v}_{1}^{i}$. In general, the closure for a combined set of $\boldsymbol{n}$ properties $\left(\boldsymbol{v}_{1}, \boldsymbol{v}_{2}, \ldots, \boldsymbol{v}_{n}\right)$ is an $n$-dimensional volume found by executing an LP problem extremizing $\boldsymbol{v}_{n}$ at a set of discrete points $\left(\boldsymbol{v}_{1}^{i}, \boldsymbol{v}_{2}^{j}, \ldots, \boldsymbol{v}_{n-1}^{l}\right)$ in the closure area of $\left(\boldsymbol{v}_{1}, \boldsymbol{v}_{2}, \ldots, \boldsymbol{v}_{n-1}\right)$. The corresponding LP problem for minimizing $\boldsymbol{v}_{n}$ is written next:

$$
\begin{aligned}
\min _{A} \boldsymbol{v}_{n} & =\boldsymbol{p}_{n}^{T} \boldsymbol{A} \text { satisfying the constraints } \\
\boldsymbol{q}^{T} \boldsymbol{A} & =1 \\
\boldsymbol{A} & >0 \\
\boldsymbol{p}_{1}^{T} \boldsymbol{A} & =\boldsymbol{v}_{1}^{i} \\
\boldsymbol{p}_{2}^{T} \boldsymbol{A} & =\boldsymbol{v}_{2}^{j} \\
& \cdots \\
\boldsymbol{p}_{n-1}^{T} \boldsymbol{A} & =\boldsymbol{v}_{n-1}^{l}
\end{aligned}
$$

To maximize $\boldsymbol{v}_{n}$, another similar problem is executed where the objective is changed as $\min _{A} \boldsymbol{v}_{n}=-\boldsymbol{p}_{n}^{T} \boldsymbol{A}$. The closure represents the range of properties obtainable when using the homogenization methodology.

The domain boundaries are computed using both upper-bound and lower-bound approaches. Upper-bound approach, which is based on the constant-strain assumption through plate thickness, computes the properties in $C$ and $\alpha$ space (both will be named as $C$ space). However, lower-bound approach, which is based on the constant-stress assumption through plate thickness, computes the properties in $C^{-1}$ and $\alpha^{-1}$ space (both will be named as $C^{-1}$ space). The experimental results can lie in any of these spaces, and so the optimum solution should be searched using both approaches. The computations of the design variables, which are determined as volume-averaged stiffness and thermal expansion quantities, are represented using an example formulation of $\langle C\rangle$. $\langle C\rangle$ computations with upper- and lowerbound approaches are given in Eqs. (17) and (18), respectively:

$$
\begin{gathered}
<C>=\int_{R} C \mathrm{Adv} \\
<C^{-1}>=\int_{R} C^{-1} \mathrm{Adv}
\end{gathered}
$$

Equations (17) and (18) show the linear relation between volumeaveraged macroscopic $\overline{<C}>$ and properties of each crystal. The same linear relation is valid for the other stiffness and thermal expansion parameters. Thus, the linear programming approach provides exact solutions to the problem of identifying ODF that lead to a given property.

The optimization is performed by sampling in $C$ space for upperbound approach and a sampling in $C^{-1}$ space for lower-bound approach. The direct linear solver determines the corresponding ODFs for the optimum properties in $C$ and $C^{-1}$ spaces. The solver is also capable of finding multiple/infinite solutions because it uses null space of the coefficient matrix to find the directions of the solutions. The use of the null-space approach requires any one solution to the problem. This one solution is obtained by sampling in the property space and using linear programming. The remaining infinite solutions are defined as the sums of this one solution and solution directions represented by null space vectors. The coefficient matrix can be defined using the linear relations for macroproperties and the unity constraint for the ODF. The size of the coefficient matrix is $13 \times 50$ for mesh 1 and $13 \times 388$ for mesh 2 because the rows are representing 12 independent linear equations for 12 parameters $(C$ and $\alpha$ elements) of the reduced space and one design constraint. The infinite solutions can be represented as shown next:

$$
\begin{gathered}
X_{i}=X_{1}+\lambda V_{i}, \quad \text { where } i=1,2,3,4, \ldots, n \\
V_{i}=\operatorname{Null}(C(:, i))
\end{gathered}
$$

where Eq. (19) defines the infinite solutions $X_{i}$ using one solution $X_{1}$ and null space vectors $V_{i} . n$ is the number of null space vectors. Even though the number of null space vectors is finite, the number of solutions are infinite because $\lambda$ can be any number that satisfies the ODF positiveness constraint $(A \geq 0)$. Because the optimization problem is solved in macroproperties' space (property closure), and the macroproperties' space is generated by the ODF values through averaging equations, any point inside this solution domain corresponds to a known set of ODF values. Therefore, there is always at least one optimal ODF solution inside this domain. The solution strategy aims to find this optimum solution not only when it is unique but also when it is multiple. 


\section{E. Optimization Results of Analytical Solution for Simply Supported} Plate

The optimization problem aims to maximize the critical increase in temperature by optimizing the ODF values, and it uses the analytical solution of a simply supported plate. The same material (HCP titanium) is considered. The optimization is performed in the reduced property space using the given procedure. 10,000 design samples are generated with Latin hypercube sampling (LHS) in the reduced space ( $C$ and $\alpha$ for upper-bound approach, and $C^{-1}$ and $\alpha^{-1}$ for lowerbound approach). The LHS method was preferred because it defines a controlled random distribution and gives information about all design space. It is also very fast to implement to the problem. The optimum parameters that provide the maximum $\Delta T_{\mathrm{cr}}$ are transformed back to the ODF space with the linear solver. The numerical approach presented to solve for multiple solutions found only one solution for this buckling problem, and this optimum result is a single crystal with a $23.0 \%$ increase for mesh 1 and a $28.3 \%$ increase for mesh 2 in the critical buckling temperature for the plate with four sides simply supported over a randomly oriented design. The optimum results obtained with the linear solver procedure are also checked with a global approach. The global approach uses the sampling in the ODF space and then uses a genetic algorithm to find the optimum solution. The genetic algorithm uses the sampling points as initial design points to start the optimization iterations. LHS was also used for sampling in the global method together with the well-known genetic algorithm, nondominated sorting genetic algorithm (NSGA-II). NSGA-II is selected as the optimization method because it is known as a fast and elitist genetic algorithm, and it always converges to the global solution [15]. The biggest drawback of gradient-based optimization algorithms is the possibility of converging to one of the local solutions instead of the global solution. For this reason, they are also strictly dependent on the initialization values of the input variables. The problems of interest in this work could potentially have many local solutions (either single-crystal solutions or combinations of polycrystal solutions). However, the final solution of interest should be the global optimum solution that gives the maximum $\Delta T_{\mathrm{cr}}$ value. To avoid any local optimum solution, a gradient-based optimization algorithm was not chosen. NSGA-II can be used for single- and multi-objective optimization problems because it has the capability of finding global optimum solutions not only in singleobjective problems but also in multi-objective problems [16]. Thus, the same optimization algorithm can also be used to solve similar problems with multiple objectives. The optimization information for the global approach is shown in Table 1 .

The global optimization approach also obtained the same optimum solutions, single crystals, that were already found with the linear solver approach. The main advantage of the linear solver approach is that it was much more efficient in terms of computation time compared to the global approach. The computational times spent on the same platform for the linear solver and global approaches are compared in Table 2 .

The optimization results are shown and compared to the randomly oriented designs in Table 3. The optimum microstructure consists of single crystals in both cases, the final optimum structure is shown in Fig. 4 for mesh 1. Figures $\underline{5}$ and $\underline{6}$ illustrate $<001>$ and $<010\rangle$ pole figures for mesh 1 . Figure $\overline{7}$ shows the final optimum microstructure of mesh 2, and Fig. $\underline{8}$ illustrates $<001>$ pole figure for mesh 2 . Figures 9 and 10 show the property closures in $C$ (upper-bound approach) and $\overline{C^{-1}}$ (lower-bound approach) spaces, respectively, for $C_{11}, C_{12}$, and $C_{22}$ parameters and their inverses. The boundaries of

Table 1 Optimization information for global solution approach

\begin{tabular}{lcc}
\hline \hline Optimization information & Mesh 1 & Mesh 2 \\
\hline Optimization algorithm & NSGA-II & NSGA-II \\
Number of number of design of experiments & 100 & 100 \\
Sampling algorithm & LHS & LHS \\
Number of total designs & 10,000 & 10,000 \\
\hline \hline
\end{tabular}

Table 2 Computational time comparison for linear solver and global optimization approaches

\begin{tabular}{lcc}
\hline \hline Method & Mesh & Computational time \\
\hline Linear solver & Mesh 1 & $\sim 27 \mathrm{~min}$ \\
Linear solver & Mesh 2 & $\sim 1.5 \mathrm{~h}$ \\
Global optimization & Mesh 1 & $\sim 9.5 \mathrm{~h}$ \\
Global optimization & Mesh 2 & $\sim 11 \mathrm{~h}$ \\
\hline \hline
\end{tabular}

Table 3 Results for a simply supported rectangular HCP titanium plate

\begin{tabular}{lc}
\hline \hline Parameter & Alpha-Ti, four sides simply supported \\
\hline Average $\Delta T_{\mathrm{cr}}($ mesh 1$)$ & $82.5, \mathrm{~K}$ \\
Average $\Delta T_{\mathrm{cr}}($ mesh 2$)$ & $82.0, \mathrm{~K}$ \\
Maximum $\Delta T_{\mathrm{cr}}($ mesh 1$)$ & $101.5, \mathrm{~K}$ \\
Maximum $\Delta T_{\mathrm{cr}}$ (mesh 2) & $105.2, \mathrm{~K}$ \\
\hline \hline
\end{tabular}

the closure triangles are determined by single-crystal solutions, and the optimum single-crystal solution is also marked in the plots.

\section{Optimization of Galfenol Beam Microstructure for Vibration Tuning}

The linear solution methodology presented in the previous sections is extended to the plastic properties to explore the microstructure design of a cantilevered Galfenol beam for a vibration-tuning problem with yielding objective (Fig. 11). Introduction of the yielding objective to the problem provides multiple solutions; however, the vibration tuning restricts these solutions to have a finite number of directions in the solution space. The modeling of the microstructure part is the same because stiffness parameters and yield stress are represented using independent ODF values. However, the number of independent ODF values is 76 at this time because Galfenol has a body-centered cubic structure. The design objective is yield stress, whereas the first bending and torsional natural frequencies are constrained for vibration tuning. The main goal of the problem is to find the best microstructure design that maximizes the yield stress of the beam and satisfies the given vibration constraints.

According to the coordinate system introduced in Fig. 11, the analytical equations of the first torsional and bending natural frequencies for an orthotropic material can be shown respectively as follows: $\omega_{1 t}=\frac{\pi}{2 L} \sqrt{\frac{G_{12} L}{\rho I_{p}}}$

$$
\omega_{1 b}=(\alpha L)^{2} \sqrt{\frac{E_{1} I_{1}}{m L^{4}}} \quad \text { and } \quad \alpha L=1.87510
$$

where $G_{12}=1 / S_{66}, E_{1}=1 / S_{11}$, and $S$ represents the compliance elements $\left(S=C^{-1}\right)$. In these formulations, $J$ is the torsion constant, $\rho$ is the density, $I_{p}$ is the polar inertia moment, $m$ is the unit mass, $L$ is the length of the beam, and $I_{1}$ is the moment of inertia along axis 1 . The computations of the yield stress using upper- and lower-bound approaches are given in Eqs. (23) and (24), respectively:

$$
\begin{gathered}
<\sigma_{y}>=\int \sigma \mathrm{AdV} \\
<\sigma_{y}^{-1}>=\int \sigma^{-1} \mathrm{AdV}
\end{gathered}
$$

The mathematical formulation of the optimization problem is given next: 

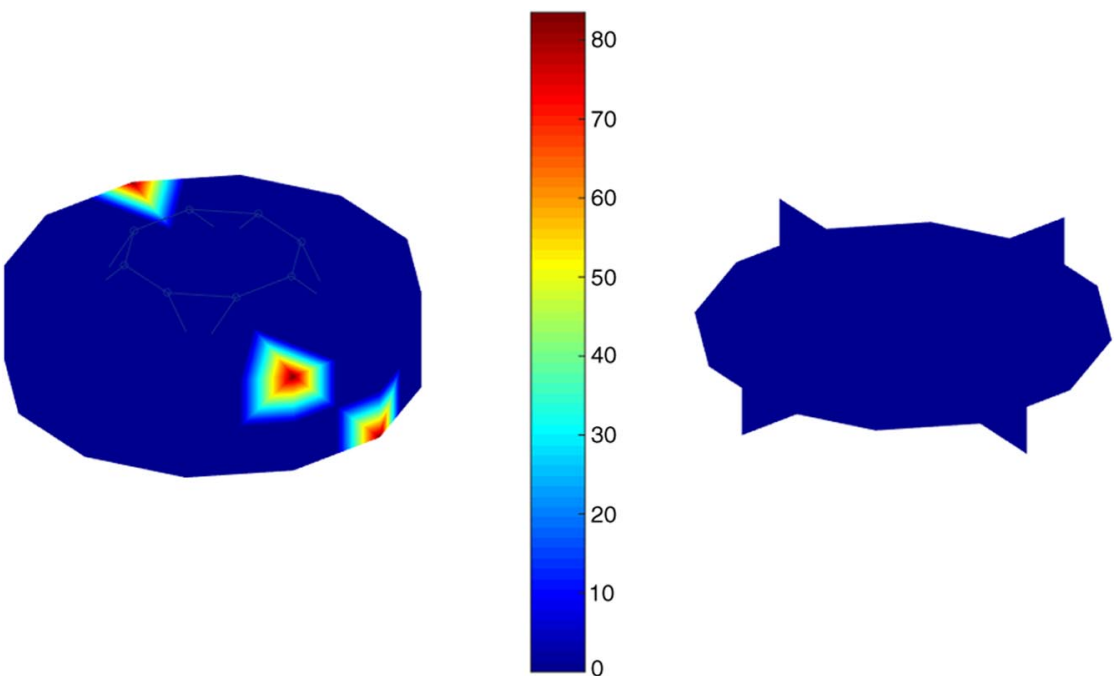

Fig. 4 Cross section of the optimal ODF for maximum critical increase in temperature (mesh 1).
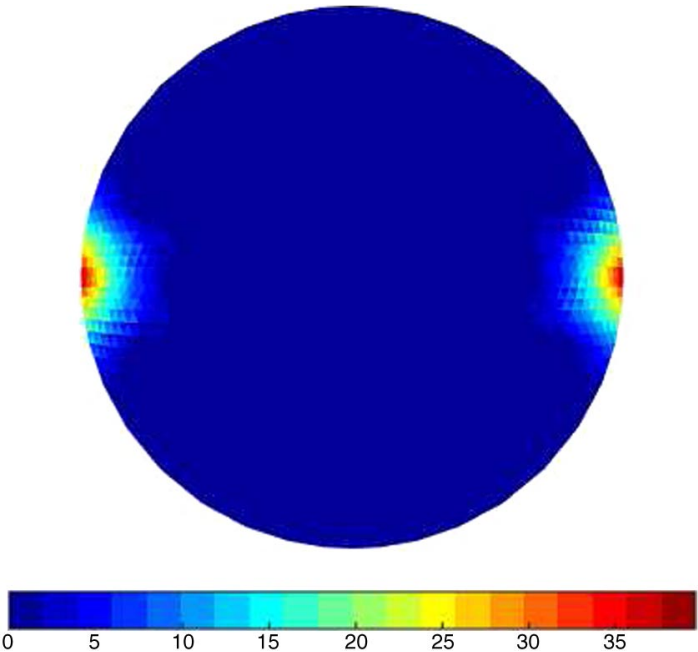

Fig. $5<001>$ pole figure for mesh 1.
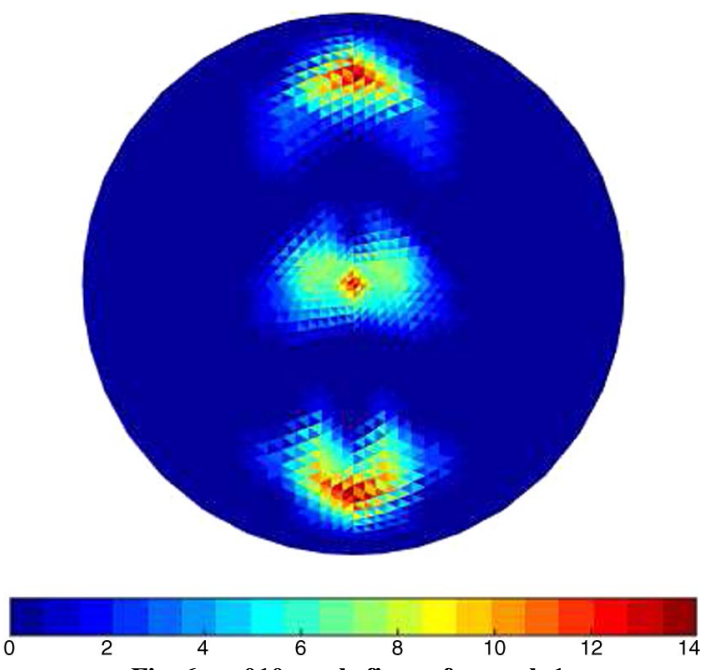

Fig. $6<010>$ pole figure for mesh 1 .

$\max \sigma_{y}$

subject to $\int \mathrm{AdV}=1$ subject to $19 \mathrm{~Hz}<=w \omega_{1 t} \leq 21 \mathrm{~Hz}$

subject to $120 \mathrm{~Hz} \leq \omega_{1 b} \leq 122.5 \mathrm{~Hz}$

$$
A \geq 0
$$

The optimization problem includes the unit volume constraint by definition as well as the constraints for the first natural frequencies to tune the beam vibration. To solve the problem, the length of the beam is taken as $L=0.45 \mathrm{~m}$, and the beam is considered to have a rectangular cross section with dimensions $a=20 \mathrm{~mm}$ and $b=3 \mathrm{~mm}$. The steps taken to optimize the microstructure are summarized next.

1) The solution space is first reduced to the independent macroelements' space consisting of microstructure-dependent properties. The limits for microstructure-dependent properties are computed using lower- or upper-bound approaches. In this problem, the microstructure-dependent properties are $E_{1}, G_{12}$, and $\sigma_{y}$.

2) One solution of the problem should be computed to start the algorithm. The solution technique depends on whether the problem is linear or not. For a linear problem, "one solution" can be computed solving a linear programming (LP) problem. However, sampling can be performed to find one solution of a nonlinear problem. The values of the microstructure-dependent input parameters will be the same in all solutions if multiple solutions exist.

3) Provided the same microstructure-dependent property values, independent solution directions are computed using the null-space approach of the linear solver. For a single solution problem, there is no existing solution direction because the single solution defines a point in the solution space.

4) In case of having multiple solutions, these solutions are computed using one solution of the problem and the independent solution directions [Eq. (20)].

The optimization problem of Galfenol beam vibration tuning has linear design objective and constraints. Therefore, the one solution to the problem could be found by solving an LP problem directly. The multiple solutions of this problem correspond to the designs having the same values for microstructure-dependent input parameters $\left(E_{1}\right.$ and $G_{12}$ ). The problem has 73 solution directions (76 optimization variables, three linear equations: two of them are for computation of $E_{1}$ and $G_{12}$, and one of them is for unit volume constraint), and these solutions are polycrystal designs. The property closure graph for $E_{1}$ and $G_{12}$ variables ( $E-G$ space) is given in Fig. 12. The parameters of the multiple optimum solution are given and compared to the best single-crystal solution in Table $\underline{4}$. Some of the optimum 


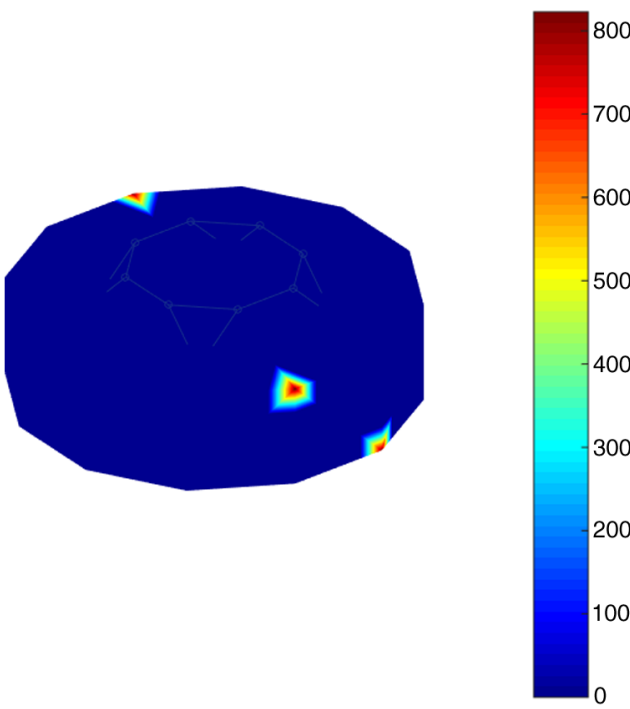

800

700

600

500

400

300

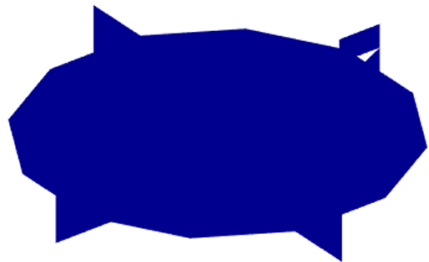

Fig. 7 Cross section of the optimal ODF for maximum critical increase in temperature (mesh 2).
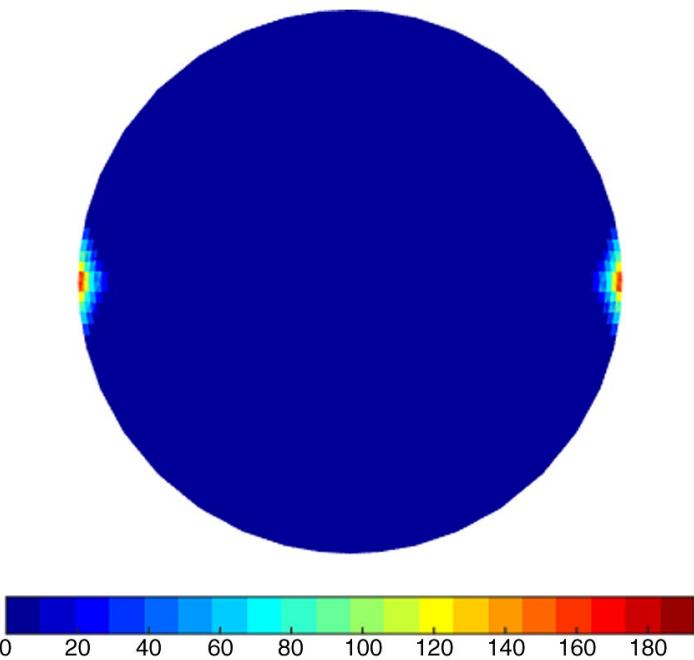

Fig. $8<001>$ pole figure for mesh 2 .

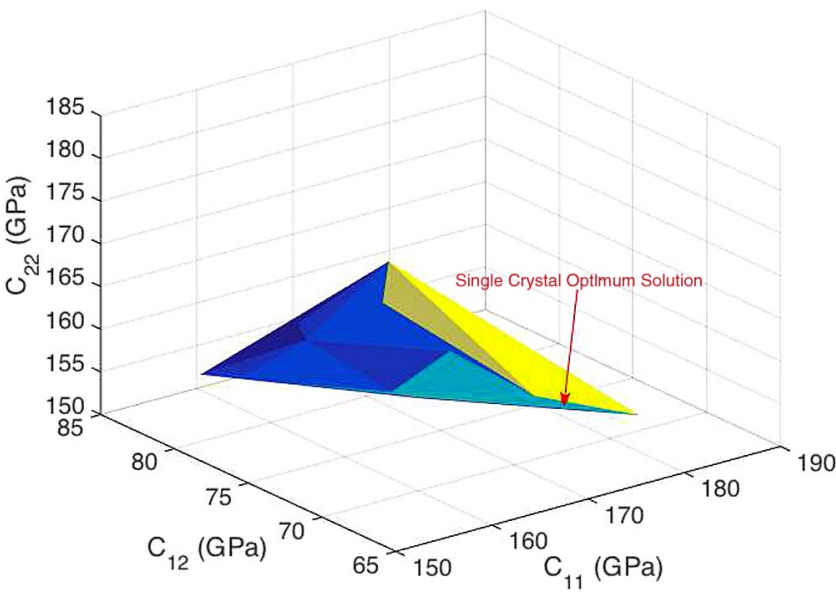

Fig. 9 Property closures in $C$ space for $C_{11}, C_{12}$, and $C_{22}$ parameters of HCP titanium.

microstructure designs are shown in Fig. 13. Because the linear solver was able to compute independent solution directions for the Galfenol beam optimization problem, each design in Fig. 13 is different from the others and has different ODF values. However, they still provide

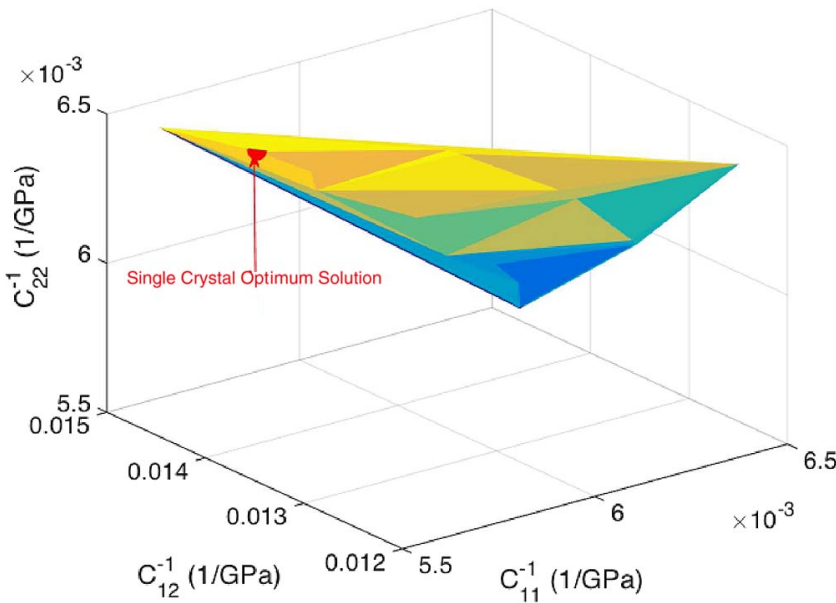

Fig. 10 Property closures for $C^{-1}$ space for $C_{11}^{-1}, C_{12}^{-1}$, and $C_{22}^{-1}$ parameters of HCP titanium.
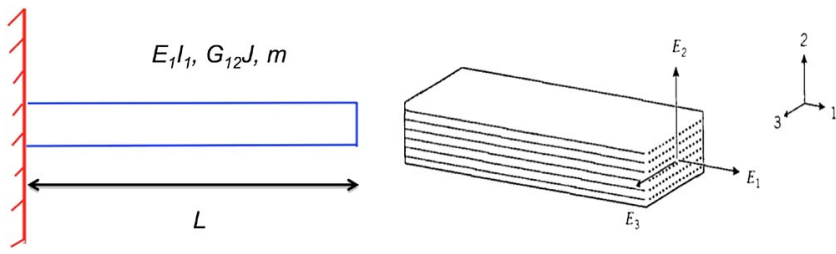

Fig. 11 Geometric representation of Galfenol beam vibration problem.

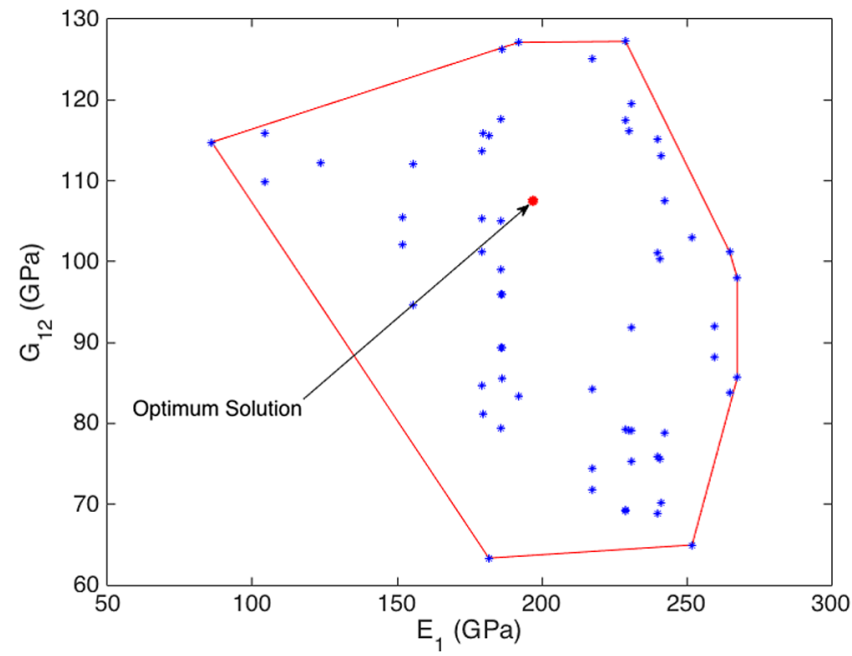

Fig. 12 Property closure for $E_{1}$ and $G_{12}$. 

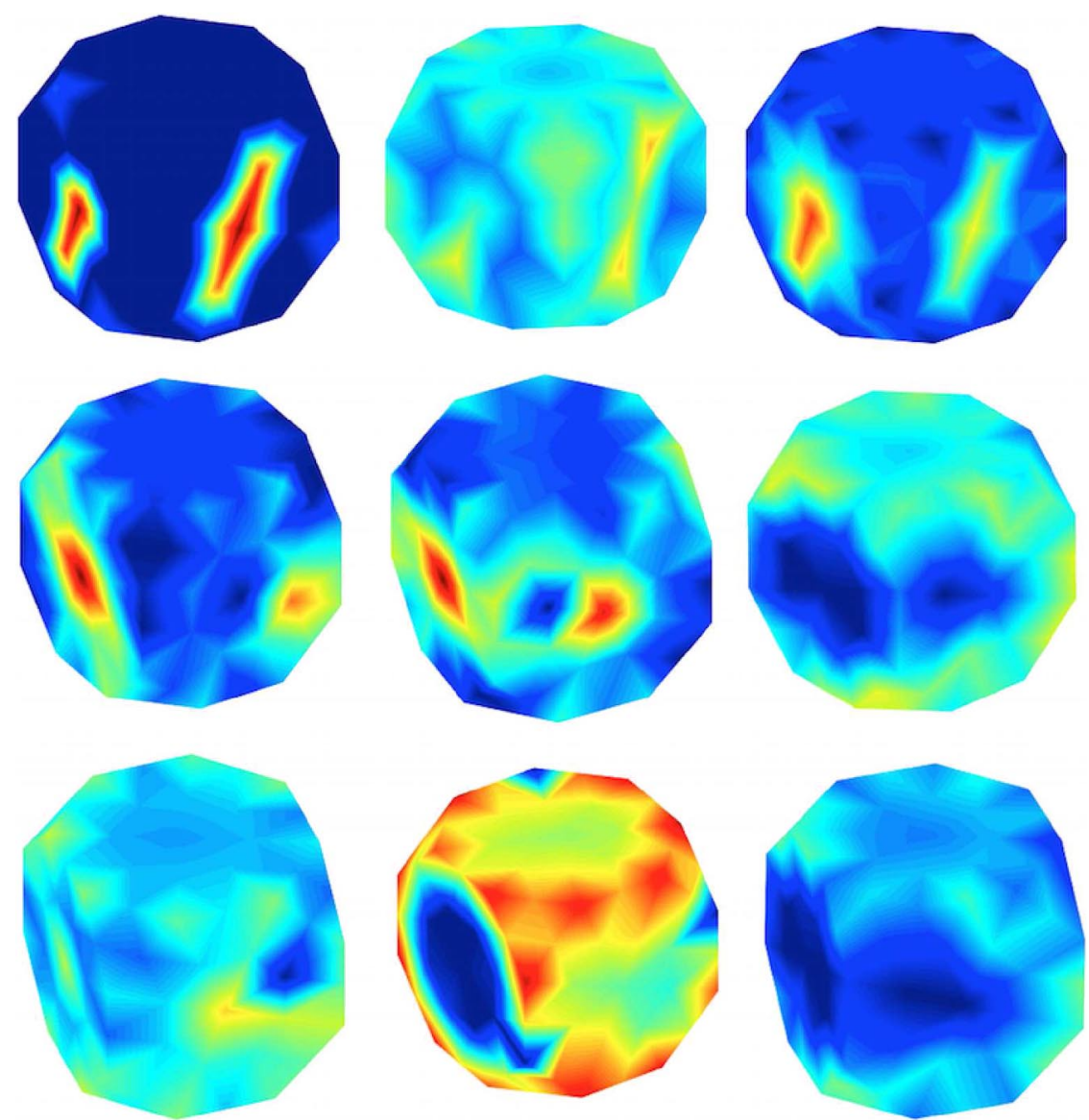

Fig. 13 Optimal ODF examples for Galfenol beam microstructure.

Table 4 Optimization results for vibration tuning of the Galfenol beam

\begin{tabular}{lcc}
\hline \hline Parameters & Optimum polycrystal & Best single crystal \\
\hline$\sigma_{y}, \mathrm{MPa}$ & 384.1256 & 352.7132 \\
$E_{1}, \mathrm{GPa}$ & 210.2585 & 215.2910 \\
$G_{12}, \mathrm{GPa}$ & 78.4528 & 78.0022 \\
\hline \hline
\end{tabular}

an identical maximum yield stress value and satisfy the design constraints.

\section{Conclusions}

This paper addresses an optimization methodology for structural problems with various macrodesign objectives. Optimization is performed in a reduced space composed of thermoelastic properties, and the optimal ODF is solved with a linear programming solver. The optimization result was also checked with a global methodology that used a genetic algorithm together with a sampling in the ODF space. Both solutions gave the same optimum solution. When compared to an HCP titanium plate with a randomly oriented microstructure, the optimal ODF provided significant 23.0 and $28.3 \%$ increases in the analytical solution, which were found in a fraction of the time of the global solution approach. For the next step, the methodology was extended to plastic properties to explore design of structural problems with a yielding objective. For this purpose, a vibration-tuning problem subjected to a maximum yield stress objective was optimized. Imposing a controlled vibration response to the problem leads multiple solutions, and these solutions are computed using the direct linear solution approach in property space. The optimal ODF distribution of these multiple solutions provided an $8.91 \%$ increase in maximum yield stress objective compared to the best single-crystal solution of the problem. It is shown that multiple solutions can be obtained by augmenting the solutions with the null space. From among these solutions, the microstructure that can be manufactured with the most economical processing route can be identified. For future work, instead of using a one-point descriptor for microstructure design (ODF), a higher-order descriptor (such as the orientation correlation function) will be used to better represent the microstructure.

\section{Acknowledgments}

This work was supported by the U.S. Air Force Office of Scientific Research, U.S. Department of Defense award FA9550-12-1-0458, and National Science Foundation award 0954390.

\section{References}

[1] Timoshenko, S. P., and Gere, J. M., Theory of Elastic Stability, 2nd ed., McGraw-Hill, New York, 1961.

[2] Bunge, H. J., Texture Analysis in Materials Science, Butterworths, London, 1982.

[3] Kocks, U. F., Tom, C. N., and Wenk, H. R., Texture and Anisotropy, Cambridge Univ. Press, Cambridge, England, U.K., 2000.

[4] Heinz, A., and Neumann, P., "Representation of Orientation and Disorientation Data for Cubic, Hexagonal, Tetragonal and Orthorhombic Crystals," Acta Crystallographica, Vol. 47, No. 6, 1991, pp. 780789.

doi:10.1107/S0108767391006864

[5] Adams, B. L., Henrie, A., Henrie, B., Lyon, M., Kalidindi, S. R., and Garmestani, H., "Microstructure-Sensitive Design of a Compliant Beam," Journal of Mechanics and Physics of Solids, Vol. 49, No. 8, 2001, pp. 1639-1663. doi:10.1016/S0022-5096(01)00016-3

[6] Kalidindi, S. R., Houskamp, J., Lyons, M., and Adams, B. L., "Microstructure Sensitive Design of an Orthotropic Plate Subjected to Tensile Load," International Journal of Plasticity, Vol. 20, Nos. 8-9, 2004, pp. 1561-1575. doi:10.1016/j.ijplas.2003.11.007

[7] Kumar, A., and Dawson, P. R., "Computational Modeling of F.C.C. Deformation Textures over Rodrigues' Space," Acta Materialia, Vol. 48, 
No. 10,2000 , pp. $2719-2736$. doi:10.1016/S1359-6454(00)00044-6

[8] Kumar, A., and Dawson, P. R., "Modeling Crystallographic Texture Evolution with Finite Elements over Neo-Eulerian Orientation Spaces," Computational Methods in Applied Mechanics and Engineering, Vol. 153, Nos. 3-4, 2000, pp. 259-302. doi:10.1016/S0045-7825(97)00072-8

[9] Liu, R., Kumar, A., Chen, Z., Agrawal, A., Sundararaghavan, V., and Choudhary, A., "A Predictive Machine Learning Approach for Microstructure Optimization and Materials Design," Nature Scientific Reports, Vol. 5, June 2015, Paper 11551. doi:10.1038/srep11551

[10] Fast, T., Knezevic, M., and Kalidindi, S. R., "Application of Microstructure Sensitive Design to Structural Components Produced from Hexagonal Polycrystalline Metals," Computational Materials Science, Vol. 43, No. 2, 2008, pp. 374-383. doi:10.1016/j.commatsci.2007.12.002

[11] Lekhnitskii, S. G., Anisotropic Plates, Gordon and Breach, New York, 1968.

[12] Taylor, G. I., "Plastic Strain in Metals," Journal of the Institute of Metals, Vol. 62, May 1938, pp. 307-324.
[13] Wenk, H. R., Preferred Orientation in Deformed Metals and Rocks, Academic Press, London, 1985.

[14] Sundararaghavan, V., and Zabaras, N., "Linear Analysis of TextureProperty Relationships Using Process-Based Representations of Rodrigues Space," Acta Materialia, Vol. 55, No. 5, 2007, pp. 1573 1587. doi:10.1016/j.actamat.2006.10.019

[15] Deb, K., Pratap, A., Agarwal, S., and Meyarivan, T., "A Fast Elitist Multiobjective Genetic Algorithm: NSGA-II," IEEE Transactions on Evolutionary Computation, Vol. 6, No. 2, 2002, pp. 182-197. doi: $10.1109 / 4235.996017$

[16] Lin, C.-H., and Lin, P.-L., "A New Non-Dominated Sorting Genetic Algorithm for Multi-Objective Optimization," Modeling Simulation and Optimization-Focus on Applications, edited by Cakaj, S., InTech, 2010.

doi: $10.5772 / 8961$
R. Ohayon Associate Editor 